
online version of the article only. Distribution for non-commercial purposes only.

\title{
Primary Malignant Melanoma of the Esophagus
}

\author{
Oya Yonal ${ }^{a} \quad$ Duygu Ibrisim $^{b} \quad$ Yıldıran Songur ${ }^{a} \quad$ Yılmaz Cakaloglu ${ }^{a}$ \\ Koray Tuncer $^{a}$ Hale Kırımlıoglu ${ }^{c}$ Sadakat Ozdil ${ }^{b}$ \\ ${ }^{a}$ Department of Gastroenterology, Memorial Şişli Hospital, ${ }^{b}$ Department of \\ Gastroenterology, Memorial Atasehir Hospital, and ' Department of Pathology, \\ Acibadem University School of Medicine, Istanbul, Turkey
}

\section{Key Words}

Endoscopic features · Endoscopic ultrasonography · Esophagus · Malignant melanoma · Treatment

\begin{abstract}
Primary malignant melanoma of the esophagus (PMME) comprises only $0.1-0.2 \%$ of all malignant esophageal tumors. PMME tumors are highly aggressive and metastasize early via hematogenic and lymphatic pathways. Treatment outcome is poor because the cancer has often advanced at the time of diagnosis. Inoperability, unsuccessful treatment with radiotherapy and chemotherapy in advanced tumors and metastases have contributed to its poor prognosis. Here, we present the endoscopic features, endoscopic ultrasonography findings and management of a PMME case.
\end{abstract}

(c) 2013 S. Karger AG, Basel

\section{Introduction}

Melanomas may arise from the mucosal epithelium lining the respiratory, alimentary and genitourinary tracts as well as the skin, all tissues that contain melanocytes. Mucosal melanomas are rare and account for $\sim 1 \%$ of all melanomas. They generally carry poorer prognosis than those arising from cutaneous sites and arise primarily in the head and neck as well as the anorectal and vulvovaginal regions. Mucosal melanomas of the head and neck commonly occur in the nasal cavity, paranasal sinuses, oral cavity and less commonly in the esophagus [1].

Primary malignant melanoma of the esophagus (PMME) comprises only $0.1-0.2 \%$ of all malignant esophageal tumors [2]. In 2001, it was estimated that only 337 cases had been

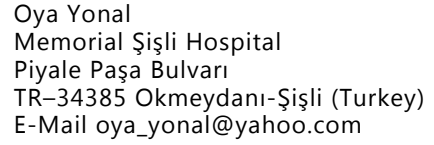


reported globally since this condition became an established clinical entity in 1963 [3]. Here, we present a case of PMME and review the literature.

\section{Case Report}

An 89-year-old female was admitted to hospital with progressively worsening dysphagia for solids during the previous month. Physical examination revealed cachexia and paleness. Apart from a mild normocytic anemia, blood tests were within the normal range. Her medical history included arrhythmia and coronary artery stent placement within the past 7 years. Upper gastrointestinal endoscopy revealed a polypoid ulcerated tumor with purple pigmentation and vascular appearance protruding into the esophageal lumen, located 27$35 \mathrm{~cm}$ from the incisors (fig. 1). Due to its vascular appearance, endoscopic biopsy was not initially performed; however, endoscopic ultrasonography (EUS) demonstrated an iso-hypoechoic, heterogeneous solid tumor mass, including cystic-anechoic areas $5 \mathrm{~cm}$ in diameter, in the esophageal wall. Doppler ultrasonography showed that the lesion was not a vascular mass but contained a few intratumoral vascular flows towards the periphery of the lesion. EUS demonstrated that the tumor had not penetrated the adventitia of the esophageal wall. Although the tumor was adjacent to large vessels and the left atrium, tumor features were not found in the vessel, and the interface echo was preserved between the tumor and the vessels (fig. 2). At the time of EUS, multiple endoscopic forceps biopsies were obtained. Diagnosis was based on histologic and immunohistochemical examinations. Histologically, there was infiltration of atypical, pleomorphic fusiform cell in the submucosa. Some of the samples had an ulcerated surface, while in others hyperplasia in the squamous mucosa was noted but intraepithelial spread was not seen. Upon immunohistochemical examination, positive diffuse S-100, melan-A and human melanoma black (HMB-45) reactions were detected as melanoma determinants. The samples were also positive for CD117, a prognostic marker of malignant melanoma (fig. 3). Thoracic and abdominal computed tomography showed no metastatic disease. Because of her debilitated condition and comorbidities, radical surgery was not considered. A metallic esophageal stent was placed to facilitate feeding.

During the first month, dysphagia was partially relieved. However, after 1.5 months of progressively worsening dysphagia and repetitive hematemesis and blood-tinged vomitus, upper gastrointestinal endoscopy was performed, revealing stent occlusion as a result of tumor overgrowth. The tumor was fragile and bled easily. There was no hemodynamic instability or severe anemia related to repetitive bleeding, and barium contrast did not pass beyond the lesion. A percutaneous endoscopic gastrostomy tube was placed surgically for nutritional support. The patient remains under follow-up care in our department 6 months after the primary diagnosis.

\section{Discussion}

PMME is a rare disease with a dismal prognosis. It occurs predominantly in male patients 60-70 years old, although younger patients have been documented [2]. Most patients complain of dysphagia, non-specific retrosternal pain and weight loss. Occasionally hematemesis and melena, as in this patient, are observed [2]. The characteristic endoscopic finding of PMME is a pigmented polypoid tumor, rarely accompanied by ulcers. Although black pigmentation is characteristic for PMME, 10-25\% of PMMEs have various colors such as purple, brown and white, depending on the melanin quantity. Amelanotic melanoma has 
no melanin pigmentation $[4,5]$. Approximately $90 \%$ of PMME are located in the distal twothirds of the esophagus [6].

According to the diagnostic criteria of primary melanoma proposed by Allen and Spitz [7], a PMME is considered as the primary tumor if it includes the typical histologic pattern of melanoma containing melanin, if the adjacent epithelium contains melanocytes and if it arises from an area of junctional changes in the squamous epithelium. Immunohistochemical staining with S-100 or HMB-45 allows accurate diagnosis of malignant melanoma [8]. In the case presented here, the presence of focal contiguous junctional melanocytic activity and the absence of melanoma in any other cutaneous, mucosal (oral, nasopharynx and anorectum) or ocular location upon clinical examination supported a diagnosis of PMME.

Thoracic and abdominal computed tomography, barium swallow examination, EUS and fluorodeoxyglucose positron-emission tomography are useful as staging methods $[9,10]$. EUS was first used in PMME in 1996 [10]. The tumor in this case appears as a hypoechoic mass or has mixed echogenicity with EUS. The outlook is dismal unless the lesion is limited to the esophageal wall. Hence, staging prior to extensive surgical intervention is desirable. Several cases of PMME have been investigated using EUS [11]. At diagnosis, $50 \%$ of patients present metastases to the liver (31\%), the mediastinum (29\%), the lungs (18\%) and the brain (13\%) [5]. Surgical resection is the standard, and only, treatment option that influences survival [12]. The mean survival time for PMME is approximately 10-14 months, with a 5-year survival rate of $\sim 4.5 \%$. There are few reported cases of survival for more than 5 years after surgery [13]. In some cases with inoperable obstructive large tumors, intraluminal brachytherapy, radiotherapy or insertion of a metal stent can be used to alleviate dysphagia [14]. Radiotherapy is a palliative modality reserved for patients with metastatic disease at high risk of mortality or who refuse surgery [5]. Other options for non-surgical or adjuvant treatment include chemotherapy, chemoradiotherapy, endocrinotherapy and immunotherapy. However, most of these treatment modalities are not widely accepted [15].

In conclusion, PMME is a rare and aggressive disease with a poor prognosis. Treatment recommendations for patients with PMME are based almost exclusively upon small, retrospective studies; therefore, the optimal management remains unknown. Tumor size and location, presence or absence of metastases, age and comorbidities of the patient affect the treatment modality. The only therapy option that influences survival is surgical treatment, whereas other therapies are palliative.

\section{Acknowledgement}

We would like to thank the Pathology Department of Acibadem University School of Medicine.

\section{References}

1 Chang AE, Karnell LH, Menck HR: The National Cancer Data Base report on cutaneous and noncutaneous melanoma: a summary of 84,836 cases from the past decade. The American College of Surgeons Commission on Cancer and the American Cancer Society. Cancer 1998;83:1664-1678.

2 Sabanathan S, Eng J, Pradhan GN: Primary malignant melanoma of the esophagus. Am J Gastroenterol 1989;84:1475-1481.

-3 Bisceglia M, Perri F, Tucci A, Tardio M, Panniello G, Vita G, Pasquinelli G: Primary malignant melanoma of the esophagus: a clinicopathologic study of a case with comprehensive literature review. Adv Anat Pathol 2011;18:235-252.

-4 Taniyama K, Suzuki H, Sakuramachi S, Toyoda T, Matsuda M, Tahara E: Amelanotic malignant melanoma of the esophagus: case report and review of the literature. Jpn J Clin Oncol 1990;20:286-295. 
Yonal et al.: Primary Malignant Melanoma of the Esophagus

5 Joob AW, Haines GK 3rd, Kies MS, Shields TW: Primary malignant melanoma of the esophagus. Ann Thorac Surg 1995;60:217-222.

-6 Sanchez AA, Wu TT, Prieto VG, Rashid A, Hamilton SR, Wang H: Comparison of primary and metastatic malignant melanoma of the esophagus: clinicopathologic review of 10 cases. Arch Pathol Lab Med 2008;132:1623-1629.

7 Allen AC, Spitz S: Malignant melanoma; a clinicopathological analysis of the criteria for diagnosis and prognosis. Cancer 1953;6:1-45.

-8 DeMatos P, Wolfe WG, Shea CR, Prieto VG, Seigler HF: Primary malignant melanoma of the esophagus. J Surg Oncol 1997;66:201-206.

-9 Yoo CC, Levine MS, McLarney JK, Rubesin SE, Herlinger H: Value of barium studies for predicting primary versus secondary non-Hodgkin's gastrointestinal lymphoma. Abdom Imaging 2000;25:368-372.

10 Namieno T, Koito K, Ambo T, Muraoka S, Uchino J: Primary malignant melanoma of the esophagus: diagnostic value of endoscopic ultrasonography. Am Surg 1996;62:716-718.

11 Holck S, Siemsen M, Jensen DB, Mogensen AM: Endoscopic ultrasonography-guided fine needle aspiration biopsy for staging malignant melanoma of the esophagus. A case report. Acta Cytol 2002;46:744-748.

12 Cheung MC, Perez EA, Molina MA, Jin X, Gutierrez JC, Franceschi D, Livingstone AS, Koniaris LG: Defining the role of surgery for primary gastrointestinal tract melanoma. J Gastrointest Surg 2008;12:731-738.

13 Gupta V, Kochhar R, Sinha SK, Das A: Primary malignant melanoma of the esophagus: long-term survival after radical resection. J Thorac Oncol 2009;4:1180-1182.

14 Kastl S, Wutke R, Czeczatka P, Hohenberger W, Horbach T: Palliation of a primary malignant melanoma of the distal esophagus by stent implantation. Report of a case. Surg Endosc 2001;15:1042-1043.

15 Kawada K, Kawano T, Nagai K, Nishikage T, Nakajima Y, Tokairin Y, Ogiya K, Tanaka K, Iwai T: Local injection of interferon beta in malignant melanoma of the esophagus as adjuvant of systemic pre- and postoperative DAV chemotherapy: case report with 7 years of long-term survival. Gastrointest Endosc 2007;66:408-410.

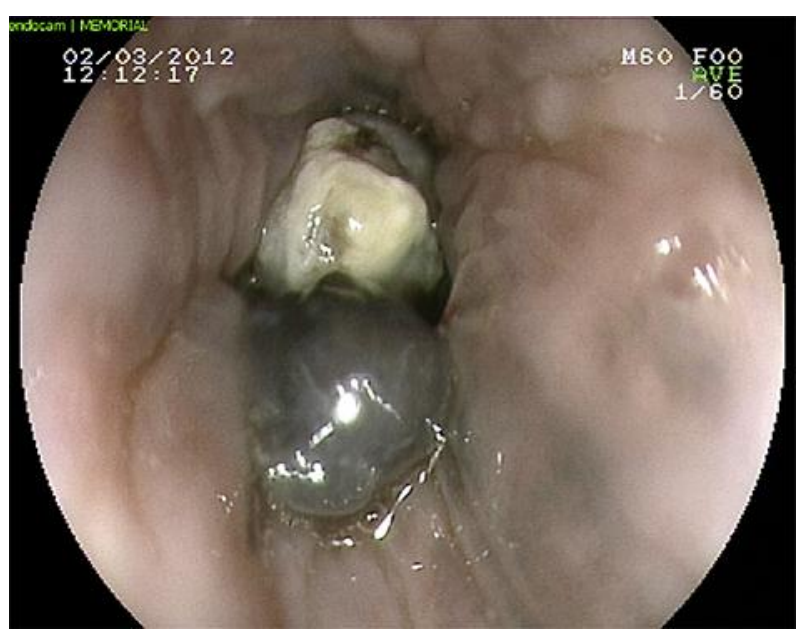

Fig. 1. Endoscopic examination showed a polypoid ulcerated tumor with purple pigmentation and vascular appearance. 
Yonal et al.: Primary Malignant Melanoma of the Esophagus

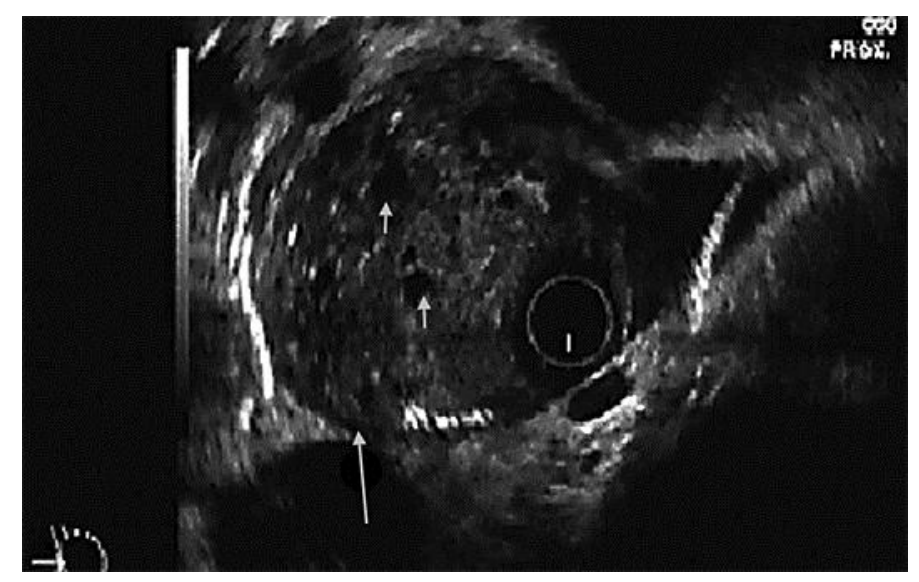

Fig. 2. Radial EUS showed an iso-hypoechoic polypoid mass lesion narrowing the esophageal lumen. The adventitia layer (long arrow) appeared to be intact and there were some intratumoral anechoic areas (short arrows).
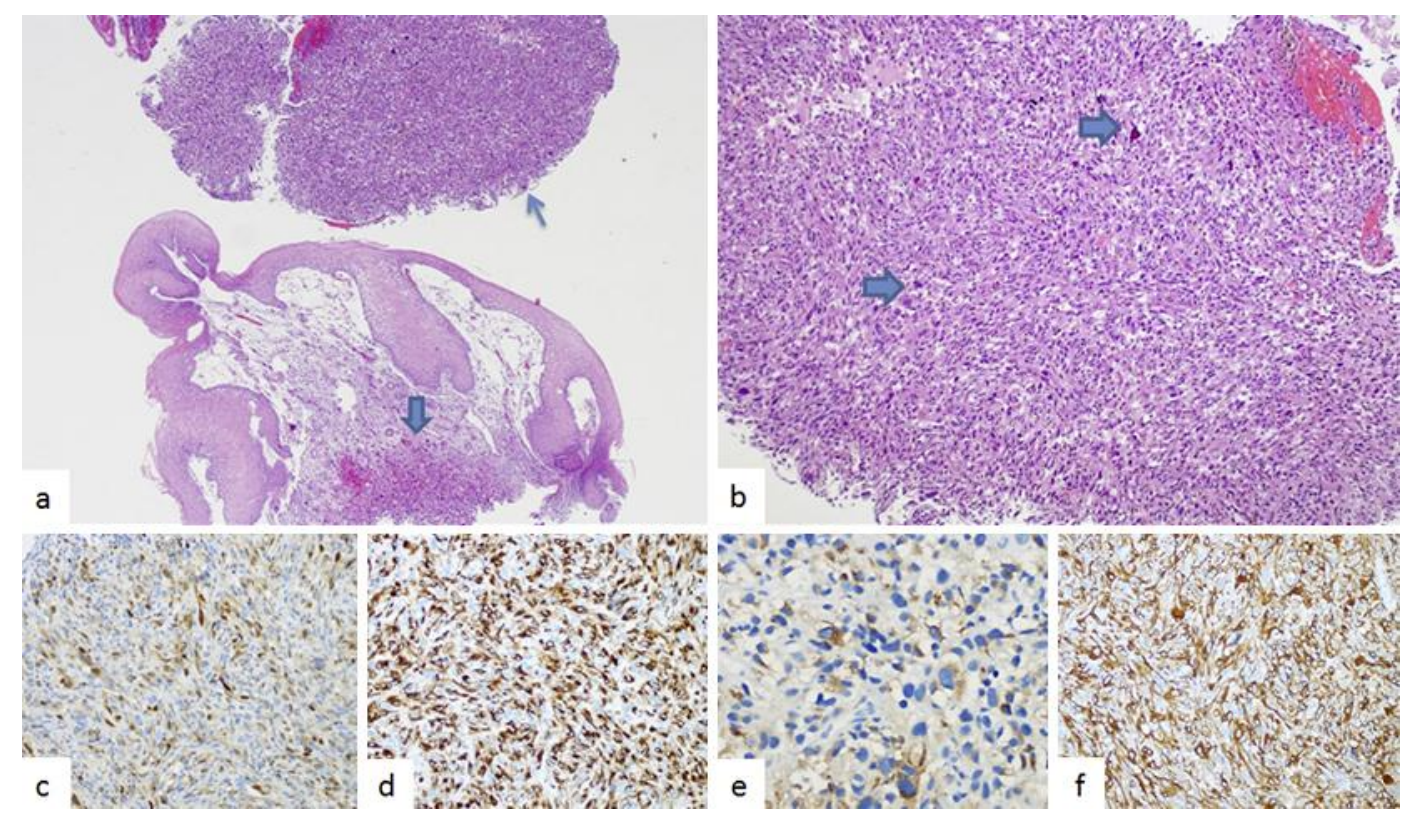

Fig. 3. a Two endoscopic biopsies, one from the ulcerated tumor (thin arrow) and the other containing subepithelial tumoral infiltration and epithelial proliferation (thick arrow) (HE, $\times 100)$. $\mathbf{b}$ The tumor was composed of spindle cells and displayed anaplastic features, including bizarre hyperchromatic cells (arrows) (HE, $\times 200$ ). Immunohistochemistry detected S-100 (c), HMB-45 (d), melan-A (e) and CD117 (f) in tumor cells $(\times 400)$. 